\title{
Historia oral y subjetividad: culturizando la democracia a partir del movimiento magisterial chiapaneco
}

\author{
Susan Street \\ CIESAS-OCCIDENTE
}

En este artículo se incursiona en la autoetnografía al revisar los tres tránsitos teórico-metodológicos por los que pasó la autora en su investigación del movimiento magisterial chiapaneco (1978-1992). A la luz de algunas categorías de la historia oral, se reflexiona sobre el impacto de la posición epistemológica de la investigadora en el sujeto-objeto del estudio y la recuperación de la memoria histórica de un movimiento social.

\section{INTRODUCCIÓN}

$\mathrm{E}$ ste breve ensayo es el producto de mi primera indagación en la historia oral como un campo problemático. A raíz de ella, tomo conciencia de que mi investigación en los últimos años tiene que ver con algunas de las inquietudes fundamentales de este campo. Tal descubrimiento a su vez me empuja a consultar mi memoria para rescatar críticamente mi trayectoria de investigación respecto al movimiento magisterial chiapaneco. Este ejercicio autorreflexivo me está dirigiendo a percibir un cambio epistemológico importante -tal vez ruptura-, momento que antes había pensado simplemente como la incorporación de métodos más cualitativos dentro de una paulatina ampliación de la sociología hacia la antropología. Mis primeras reconstrucciones desde "la historia oral" me exigen poner la subjetividad en el centro. Así estoy comprendiendo mi propia insistencia últimamente en culturizar la democracia.

En esta ponencia, contrasto dos formas de investigar el tema de los movimientos sociales y la democracia en México. Corresponden a dos mo- 
mentos históricos en mi conocimiento del movimiento magisterial chiapaneco (1979-1992): 1) el movimiento como actor político y la democracia como objetivo político estratégico (1986-1990) ${ }^{1}$ y 2) el movimiento como sujeto y la democracia como subjetividad (19911995). ${ }^{2}$ Organizo la discusión de estas etapas, o formas de acercarme a la realidad; en función de "abandonos" y replanteamientos.

Empiezo describiendo las modificaciones en mi manera de concebir el objeto de estudio: abandono el movimiento magisterial como objeto para asumirlo como sujeto. En seguida reviso las bases de mi vínculo con los maestros chiapanecos: compartir una afinidad ideológica de fines, a pensarme coconstructora de significados. Por último, reflexiono sobre mi concepto de democracia. Dejé de considerar a la democracia como un adjetivo no problematizado por los maestros ni por su intérprete, para repensarla como una experiencia vital, como una emotividad significante de "la dignidad". Asimismo, la democracia abandonó su definición formal, liberal, como una serie de procedimientos y reglas de juego, para entenderse como la afirmación de los excluidos como seres humanos con derechos (a la autodeterminación y al autogobierno).

\footnotetext{
${ }^{1}$ Street, "Lucha", 1985, pp. 183-214, y Maestros, 1992.

${ }^{2}$ Street, "Cultura", 1994, pp. 427-466; "Democracia", 1995, pp. 61-84; "Democracia", 1997.
}

\section{DE OBJETO A SUJETO.}

Pienso mi tránsito de objeto a sujeto, en abstracto, como un cambio en la formulación del quehacer de las ciencias sociales: de la objetivación del objeto, a la simultánea subjetivación del objeto y objetivación del sujeto. Esto representa dejar de pensar la práctica científica como operaciones unidimensionales, donde se construyen las relaciones sociales a partir de linealidades deducidas de la macroteoría, y en su lugar, asumir una dialéctica entre subjetivación y objetivación que significa abordar las subjetividades desde articulaciones multidimensionales (entre planos de la realidad, temporalidades y espacialidades). ${ }^{3}$

Ejemplificaré la primera perspectiva. Abordé al Movimiento Magisterial Chiapaneco (MMC) con un modelo de análisis político derivado de una teoría marxista del Estado que delimitaba el ámbito de posibilidades de las clases subordinadas a ciertos tipos de resistencia y oposición. Definí el movimiento magisterial como una fuerza que podía llegar a ser "actor político" e impactar a ciertos ámbitos burocráticos del Estado. Esencialmente, concebí al movimiento como un indicador de las fisuras y crisis en el régimen político autoritario. Lo que había que hacer era ubicar el MMC en varias "correlaciones de fuerzas" y en relación con otros actores políticos. Lo que objetivaba eran las interacciones entre

\footnotetext{
3 Para elaborar la nueva perspectiva me apoyo en la obra de Hugo Zemelman, especialmente Conocimiento, 1987, y "Esperanza", 1995, pp. 11-28.
} 
los patrimonialistas, los tecnócratas y los democráticos en su lucha por el poder (por el control de la burocracia estatal). De manera significativa, para nombrar a los actores, me basé en $\mathrm{mi}$ idea de sus propósitos respecto al Estado (conservarlo o modificarlo según determinados intereses.)

No hace falta subrayar aquí que este enfoque no me permitió conocer al MMC como sujeto. Mi explicación de su impacto en esferas externas se basó en categorías "organizativas". Así, afirmé que su "alta capacidad transformativa como movimiento" se debió a que los maestros habían creado un nuevo sistema autogestionario descentralizado basado en "el poder de las bases" que había renovado la acción sindical a nivel regional. Pero, mi "explicación", que coincidía a grandes rasgos con la de los dirigentes, era demasiado estructural; no daba cuenta de lo que había notado al practicar la "observación participativa" en las acciones e instancias colectivas del MMC (marchas, tomas de edificios, asambleas, etc.): la gran expresividad emotiva en torno a ciertos valores.

Los maestros democráticos chiapanecos se concebían de manera muy diferente de otros maestros: se afirmaban como mejores, como los más justos, como los más honestos, como los más humanos, todo ello como producto de su unión/fusión al movimiento. Yo misma había sentido el llamado de las consignas y las demandas, pues había asumido un lugar en esta comunidad. No obstante que los dirigentes manejaban definiciones formales de la democracia como procedimiento, tendian a explicar la adhe- sión de los maestros de base al movimiento, usando otro marco de referencia, el que tenía que ver con procesos de humanización. Un dirigente me dijo en 1986 que

Lo más importante es que los maestros decidan. Antes, el supervisor y el representante sindical, en mancuerna, eran quienes, por ejemplo, quitaban y daban plazas como les venía en gana. Ahora es el maestro en asamblea quien decide los criterios [...] para hacer cambios en las zonas escolares [...] Los maestros discuten sus cosas horas y horas: Nadie nos puede quitar esto [...] No es posible retroceder en el tiempo, pues el maestro ba recuperado su voz.

Yo recuerdo que me intrigaba la emotividad compartida entre los maestros. Quise entenderla de manera más profunda como una expresión del "corazón común" indígena, del ser "laján" o "lajanotik" (el concepto tojolabal de un nosotros basado en la reciprocidad entendida como mutuo apoyo y respeto y como corresponsabilidad en el trabajo colectivo). Opté por un enfoque más cultural. Concebí al movimiento como creador de nuevas identidades y recurrí a la sociología epistémica para abordar las "gramáticas de conceptualización" involucradas en formas (diversas) de pensar la democracia como experiencia. Rechacé como parcial (y sobrepolitizada) la óptica de los dirigentes, mis anteriores informantes, y me fui con la otra parte, la más silenciosa y menos capaz de teorizar: los maestros de base. Me propuse construir "el pensamiento de las bases" para entender por qué los maes- 
tros optaban por ser "bases", con cuáles inversiones emotivas lo hacían y cómo vivieron los maestros el tránsito de "masas" desorganizadas a "bases" de un nuevo sujeto.

Ahora entiendo que, al buscar conocer las lógicas atrás de las acciones y de los vínculos de los maestros, estaba tomando en serio una idea clave de la historia oral: la relevancia de la subjetividad del narrador. ${ }^{4}$

(Oral sources) tell us not just what people did, but what they wanted to do, what they believed they were doing, what they now think tbey did...what makes them unique and necessary is their plot -the way in which the narrator arranges materials in order to tell the story. The organization of the narrative (subject to rules which are mostly the result of collective elaboration) reveals a great deal of the speakers' relationship to their own bistory.

Apoyada en entrevistas con seis maestros de base en 1992, construí sus historias de participación en el MMC, con la intención de generar teoría desde abajo. ${ }^{5}$ Al buscar identificar las estructuras profundas informando las narrativas, ${ }^{6}$ pude elaborar un texto etnográfico para describir "las lógicas participativas individuales", "las prácticas significantes colectivas", y "las subjetividades sociales de las bases" del MMC, y llegué a postular que éste ofrecía dos subjetividades democráticas a

${ }^{4}$ Portelli, “Peculiarities”, 1981, pp. 96-107.

${ }^{5}$ Glaser y Strauss, Discovery, 1967.

${ }^{6} \mathrm{~A}$ posteriori puedo ver silmilitudes entre mi procedimiento analítico y lo recomendado por Ron Grele en "Listen", 1979, pp. 33-42. los maestros: la incorporativa y la interpelativa.

Los maestros que se "incorporan" al movimiento se identifican con los procedimientos de la democracia de base, los mismos que asumen como un deber del individuo acatar. Estas personas entienden la formación del consenso como la asistencia a las actividades (decididas por las mayorías) y como el ejercicio individual de cumplir con el papel asignado a las bases en un determinado esquema de la toma de decisiones sindical $[\ldots]$ Los maestros que se vinculan activamente con la modalidad interpelativa del movimiento, fortaleciéndola con su adhesión, son aquellos que afianzan su participación en las partes sustantivas de la democracia de base porque se apropian de los valores colectivos del movimiento y porque viven las prácticas de la base como un papel sustentado en una ética colectiva. Para ellos, la formación del consenso no deviene de hacer una aportación individual al constituirse en "voz y voto", sino de ser una representación ejemplar de un valor democrático o de "la identidad democrática" como un conjunto axiológico totalizador. $^{7}$

En este elaborado proceso analítico, relacioné los motivos de la acción individual (explicaciones individuales de sus vínculos con los diversos colectivos del MMC), con las prácticas deliberativas/argumentativas del MMC ("razonar entre propuestas", "denunciar abusos", "protestar la corrupción", "votar en asamblea", "vigilar el cumpli-

${ }^{7}$ Un ejemplo de mi descripción analítica de las categorías sociales de los maestros para aclatar sus conceptos de democracia. 
miento del derecho", etc.) y con la expresión más abstracta de la identidad democrática ("la ética de reciprocidad"), en diferentes momentos históricos. De esta manera desarrollé una propuesta específica para entender la subjetividad como articulaciones dialécticas entre lo individual y lo colectivo. Esto concuerda con la propuesta teórico-metodológica de Hugo Zemelman.

Se trata de recuperar el proceso social que resulta de las múltiples formas de interacción que caracterizan a la realidad como un complejo proceso de transitividades, y cuya expresión más elocuente son las distintas modalidades de prácticas que son el resultado del proceso mismo de constitución de la subjetividad social como productoras del mismo. ${ }^{8}$

En suma, creo que es importante decir aquí que ya había pasado a teorizar las subjetividades, incluyendo $m i$ participación como investigadora, en la medida que el proceso analítico de las historias de vida dependía de la explicitación de mis propias categorías y conceptos teóricos. En ese momento todavía no incluía en la teorización elementos de mi propia subjetividad; ahora entiendo que hacerlo implicaría reescribir la historia tomando en cuenta las "dialécticas de conocimiento" que unieron a la investigadora y al sujeto en su coproducción de la memoria del MMC.

'Zemelman, "Comentarios", 1994, p. 21
DE "IA POSICIÓN POLÍTICO-IDEOLÓGICA" AL "SOUL HISTORY" COMO MEDIO DE VINCUILACIÓN PERSONAL CON EL OBJETO/SUJETO

"La praxis" se ha mantenido como el criterio de verdad más importante en mi proceso de construir conocimiento. Pero el cambio en mi relación con el MMC se debe a que abandoné un entendimiento de praxis basado en un concepto de "la investigación al servicio del movimiento popular", donde el conocimiento "entregado" o "devuelto" debía enriquecer las capacidades de acción del sujeto de cambio histórico. Y empecé a entenderme a mí misma como un sujeto (auto)interpretativo, simultáneamente producto del MMC, en tanto esta comunidad me era relevante vitalmente, y productora de su democracia (o antidemocracia), en tanto "la ética de reciprocidad" me "hablaba" a mí a través de algunos valores de mi infancia. Entiendo ahora el afán mío por construir y significar su utopía social. ${ }^{10}$

En algún momento de mi investigación con los maestros chiapanecos; me había convertido en su vocera, sin haber entendido plenamente mis "proyectos inconscientes individuales" 11 transferidos a los sujetos y a las situa60.

9 Passerini, "Mythbiography", 1990, pp. 49-

${ }^{10} \mathrm{Me}$ refiero a los valores (elaborados en mis artículos) en lo que llamo su "ética de reciprocidad": respeto a sí mismo, al otro y al derecho colectivo; honestidad y responsabilidad; combatividad y solidaridad expresadas en una relación recíproca y equitativa entre dirigentes $y$ bases.

${ }^{11}$ Figlio, "Oral", 1988, pp. 120-132. 
ciones sociales. Al resignificar mi historia personal, me percaté de cómo había objetivado mi propia voz en la identificación de los procesos en los que los maestros chiapanecos "recuperaron su voz". No me refiero aquí al principio antropológico de la explicitación de la voz interrogante de la entrevistadora. Como sugiere Alessandro Portelli, ${ }^{12}$ el compromiso con este sujeto sigue siendo de naturaleza política, pero trasciende la cuestión ideológica.

Radical bistory-writing is not a matter of ideology, of subjective sides-taking on the bistorian's part, or of what kind of sources they use. It is ratber inberent in the bistorian's presence in the story being told, in the assumption of responsibility wbich inscribes bim or her in the account and reveals bistoriography as an autonomous act of narration. Political choices become less visible and vocal, but more basic.

Finalmente, $\mathrm{y}$ basándome en otro entendimiento de praxis que remite a las necesidades totales del hombre, abandoné mi papel de puente para (intentar) pasar "simplemente" a construir un pensamiento crítico, en el marco de crear la democracia.

\section{DE LA DEMOCRACIA COMO DISCURSO}

\section{A LA DEMOCRACIA COMO PRAXIS}

En este tercer cambio, abandoné una definición a priori y aproblemática de "democracia", para reelaborar otro concepto como parte de un proyecto sus-

\footnotetext{
${ }^{12}$ Portelli, "Peculiarities", 1981, p. 105.
}

tancializador de la democracia (en un doble sentido de historización y de humanización). Junto con otros seguidores de Gramsci, entiendo la democracia como la praxis activa de las clases subalternas, es decir, como la afirmación de los excluidos, marginados, explotados, oprimidos, quienes, al negar su condición de "ser negados por otros", buscan objetivarse plenamente como seres humanos. ${ }^{13}$

Esto fue posible debido a que entendí mejor las condiciones de producción del discurso democrático de los dirigentes del MMC. Pude separarlo de las maneras en que ellos asumen sus diversos papeles de ideólogos, líderes y oradores, pero también como los narradores oficiales del MMC. Al abocarme a articular las dinámicas constitutivas del sujeto en torno a la relación fundamental entre dirigentes y bases, empecé a entender los procesos de exclusión que fueron reduciendo a los participantes del MMC y desilusionando a las bases. Su memoria colectiva también registra el proceso histórico de mitificación de "la identidad democrática". Es decir, la ética de reciprocidad fue transformada en una referencia mítica e ideal y utilizada estratégica y políticamente por los maestros chiapanecos como un discurso, mas no como una praxis.

La democracia sólo existió como una praxis en momentos históricos muy precisos; durante las fases iniciales de las movilizaciones, cuando los maestros rechazaron su subordinación institucional (gremial, sindical, profe-

${ }^{13}$ Dussel, "Introducción", 1992, pp. 45-102; Heller, Historia, 1985. 


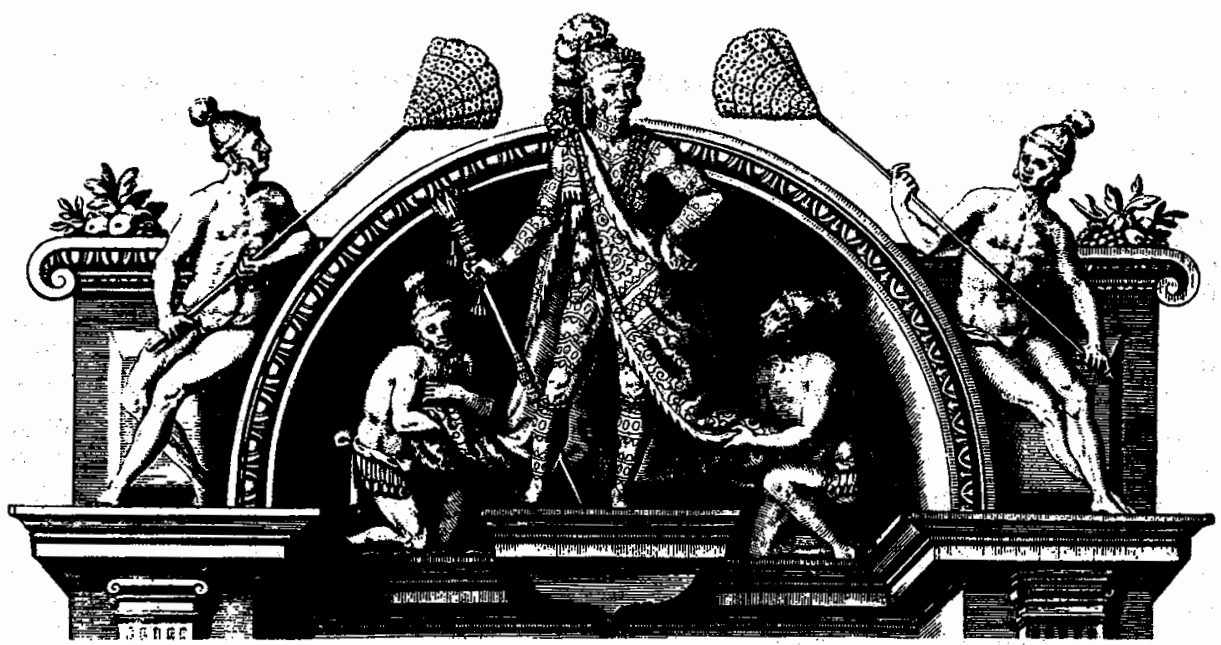

sional). Estos actos afirmativos (de reconocerse como personas con derechos), se dieron bajo dos formas y en dos momentos: como un grito de las masas (de rechazo al abuso de sus jefes, al maltrato entre maestros, a la corrupción, a la arbitrariedad, etc.) y como una voz de las bases (para expresar sus necesidades y actuar para cumplirlas).

Concluyo, entonces, que la memoria colectiva de los maestros chiapanecos está constituida con elementos de la democracia como historia, pero predominó la democracia como mito. Los maestros conocieron la democracia real en tanto experimentaron su autogobierno como una forma de vida, pero esta vivencia fue opacada al construir la democracia también como un mito.

\section{BIBLIOGRAFía}

-Dussel, Enrique, "La introducción de la transformación de la filosofía de $K$. $O$.
Apel y la filosofía de la liberación (reflexiones desde una perspectiva latinoamericana)" en Fundamentación de la ética y filosofía de la liberación, Siglo XXI, México, 1992.

-Figlio, Karl, "Oral history and the unconscious", History Workshop, núm. 26, otoño, 1988, pp. 120-132.

-Glaser, Barney G. y Anselm L. Strauss, The discovery of grounded theory: strategies for qualitative research, Aldine Publishing Company, Nueva York, 1967.

-Grele, Ron, "Listen to their voices; two case studies in the interpretation of oral history interviews", Oral History, vol. 7, núm. 1, 1979.

-Heller, Agnes, Historia y vida cotidiana: aportación a la sociología socialista, Grijalbo, 1985 (Enlace).

-Passerini, Luisa, "Mythbiography in oral history" en Raphael Samuel y Paul Thompson (coords.), The myths we live by, Routledge, Nueva York, 1990.

-Portelli, Alessandro, "The peculiarities of oral history", History Worksbop, núm. 12, otoño, 1981, pp. 96-107.

-Street, Susan, "La lucha por transformar el aparato burocrático de la SEP", Re- 
vista Mexicana de Sociología, IIS-UNAM, núm. 4, octubre-diciembre, 1985, pp. 183-214.

Maestros en movimiento; transformaciones en la burocracia estatal (1978-1982), cIBSAS, México, 1992 (Colección Miguel Othón de Mendizábal). " "La cultura política del movimiento magisterial chiapaneco" en Jorge Alonso (coord.), Cultura política y educación cívica, Miguel Ángel Porrúa, México, 1994, pp. 427-466.

"La democracia "desde abajo': construyendo 'I dignidad' a partir del movimiento magisterial chiapaneco", Espiral (Estudios sobre Estado y Sociedad), Universidad de Guadalajara, vol. 1, núm. 3, mayo-agosto, 1995, pp. 61-84.

"Democracia como reciprocidad: modalidades participativas de 'las bases' del movimiento magisterial chiapaneco", en Procesos políticos en el Mé- xico contemporáneo: una visión antropológica, INAH/Plaza y Valdez, México, 1997, en prensa.

Zemelman, Hugo, Conocimiento y sujetos sociales; contribución al estudio del presente, El Colegio de México, México, 1987.

, La esperanza como conciencia (un alegato contra el bloqueo histórico imperante: ideas sobre sujetos y lenguaje)" en Hugo Zemelman (coord.), Determinismos y alternativas en las ciencias sociales de América Latina, Centro Regional de Investigaciones Multidisciplinarias-UNAM/Editorial Nueva Sociedad, México, 1995, pp. 11-28.

"Comentarios para reiniciar el debate" en Tomás R. Villasante (coord.), Las ciudades bablan. Identidades y movimientos sociales en seis metrópolis latinoamericanas, Editorial Nueva Sociedad, Caracas, 1994. 